\title{
Technology platform for the fabrication of titanium nanostructures
}

Serge Ecoffey, Marc Guilmain, Jean-François Morissette, Frédéric Bourque, Jérémy Pont, Bruno Lee Sang, and Dominique Drouin

Citation: Journal of Vacuum Science \& Technology B, Nanotechnology and Microelectronics: Materials, Processing, Measurement, and Phenomena 29, 06FG06 (2011); doi: 10.1116/1.3657517

View online: http://dx.doi.org/10.1116/1.3657517

View Table of Contents: http://avs.scitation.org/toc/jvb/29/6

Published by the American Vacuum Society

\section{Instruments for Advanced Science}

Contact Hiden Analytical for further details: w www.HidenAnalytical.com E info@hiden.co.uk CLICK TO VIEW our product catalogue

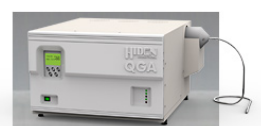

Gas Analysis

dynamic measurement of reaction gas streams , catalysis and thermal analysis

molecular beam studies

dissolved species probes

fermentation, environmental and ecological studies

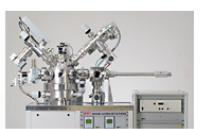

Surface Science

UHVTPD

end point detection in ion beam etch elemental imaging - surface mapping

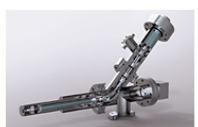

Plasma Diagnostics

' plasma source characterization

etch and deposition process reaction

, kinetic studies

analysis of neutral and radical species

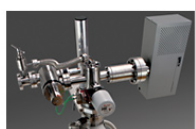

Vacuum Analysis

partial pressure measurement and contro

of process gases

- reactive soutter process control

vacuum diagnostics

- vacuum coating process monitoring 


\title{
Technology platform for the fabrication of titanium nanostructures
}

\author{
Serge Ecoffey, ${ }^{a)}$ Marc Guilmain, Jean-François Morissette, Frédéric Bourque, Jérémy Pont, \\ Bruno Lee Sang, and Dominique Drouin \\ Nanofabrication and Nanocharacterization Research Center (CRN2), Université de Sherbrooke, \\ 2500 Boulevard Université, Sherbrooke QC, J1K 2R1, Canada
}

(Received 27 June 2011; accepted 8 October 2011; published 3 November 2011)

\begin{abstract}
This paper presents two approaches for the fabrication of top-down titanium nanostructures. The first approach involves electron beam lithography followed by a tailored titanium plasma etching. The two main challenges of this process lie in the optimization of the negative tone $\mathrm{Ma}-\mathrm{N}$ electroresist resolution and in the definition of a controlled titanium etching process for titanium patterns less than $20 \mathrm{~nm}$ thick and wide. The second proposed approach is a damascene process where the titanium nanostructures are buried in the oxide. Very shallow and narrow $(20 \mathrm{~nm} \times 20 \mathrm{~nm})$ trenches are first patterned in the oxide and nanostructures are obtained by planarization of an evaporated titanium film. The dimensions of the structures are defined by the electron beam lithography resolution and the etching recipe. The third dimension is given by the titanium or any other metal thickness and can be controlled down to few nanometers thanks to the planarization step. (ㅇ 2011 American Vacuum Society. [DOI: 10.1116/1.3657517]
\end{abstract}

\section{INTRODUCTION}

Titanium oxide (rutile) has peculiar physical properties ${ }^{1}$ that have already been exploited since the sixties for the fabrication of passive devices, ${ }^{2}$ while titanium (Ti) is a bulk material for the fabrication of microstructured devices such as microelectromechanical systems ${ }^{3}$ or microbolometers. ${ }^{4}$ To date, very few results have been reported on the fabrication of titanium/rutile nanostructured devices ${ }^{5}$ although titanium/ rutile nanostructures have demonstrated their great potential for use in the fabrication of future nanoelectronic devices and circuits, ${ }^{6}$ such as MEMRISTORS, ${ }^{7}$ or single electron transistors (SETs). ${ }^{8}$

The fabrication of titanium nanostructures less than $100 \mathrm{~nm}$ requires precise control of the resist pattern transfer in the metal through the etching process. Dry etching of titanium thin films involves fluorine and/or chlorine based chemistries ${ }^{3,4,9,10}$ that lead to etch rates in the range of a $\mu \mathrm{m} /$ min. However, Gilmartin et al. ${ }^{5}$ have developed a nanoscale $\mathrm{Ti}$ etch process using electron beam lithography (EBL), achieving $70 \mathrm{~nm}$ critical dimension (CD) structures.

Chemical mechanical polishing (CMP) was first exploited in the back-end-of-line (BEOL) for multilevel metallization in the early 1990s. ${ }^{11}$ It was then introduced in the front-endof-line through shallow trench isolation technology in $1995 .^{12}$ After incremental developments mainly due to new BEOL materials it has been integrated in the $45 \mathrm{~nm}$ technology node as a solution for high K-metal gate structures. ${ }^{13}$ CMP is a promising candidate for the $32 \mathrm{~nm}$ node and future applications such as fin-shaped field effect transistor, phase change memory, 3D integration, ${ }^{12,13}$ and SETs. ${ }^{8,14}$

This paper proposes two different approaches for the fabrication of top-down titanium nanostructures. The first approach involves EBL followed by a tailored titanium plasma etching. The two main challenges of this process lie in the optimization of the negative tone $\mathrm{Ma}-\mathrm{N}$ electroresist resolution and in

${ }^{a)}$ Electronic mail: serge.ecoffey@usherbrooke.ca the definition of a controlled titanium etching process. The second approach is a damascene process where the titanium nanostructures are buried in the oxide. Very shallow and narrow trenches are first patterned in the oxide and nanostructures are achieved by planarization of the evaporated titanium film with a CMP process.

\section{FABRICATION PROCESS}

\section{A. Titanium dry etching}

The first proposed method for the fabrication of titanium nanostructures consists of EBL followed by plasma etching. The EBL was achieved using Ma-N 2401 electroresist from Micro resist technology, and a Zeiss LEO 1530 scanning electron microscope (SEM) working at $20 \mathrm{keV}$. Figure 1(a) shows an SEM image of Ma-N isolated lines on a thermally oxidized silicon substrate. The electroresist has been spun at $5000 \mathrm{rpm}$ while Ma-D 525 was used for the development. The lines are $120 \mathrm{~nm}$ wide which corresponds to the ellipsometric measurement of the resist thickness after spinning. When the width of the resist lines is decreased to less than $30 \mathrm{~nm}$, their aspect ratio is greater than a factor of four and the lines collapse [the darker lines in Fig. 1(a)]. Figure 1(b)

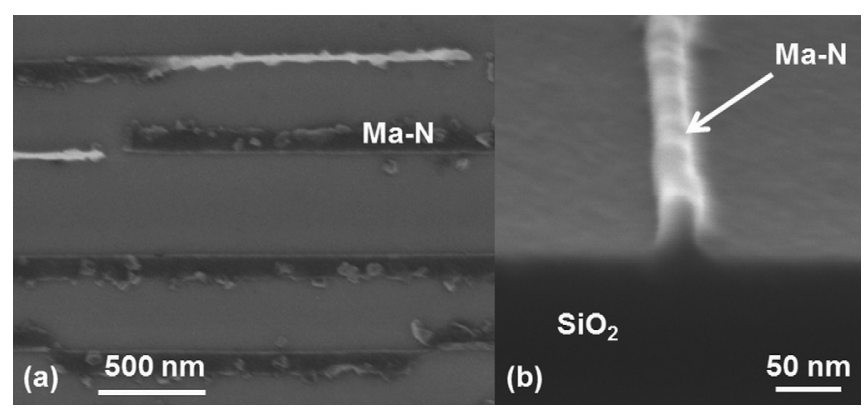

FIG. 1. SEM images of developed $\mathrm{Ma}-\mathrm{N}$ lines on $\mathrm{SiO}_{2}$ : (a) $120 \mathrm{~nm}$ collapsed lines (dark lines), together with standing lines (bright lines); (b) less than $20 \mathrm{~nm}$ thick isolated Ma-N line. 
TABLE I. Summary of the optimized plasma etching parameters.

\begin{tabular}{ccccc}
\hline \hline $\mathrm{Cl}_{2}(\mathrm{sccm})$ & $\mathrm{BCl}_{3}(\mathrm{sccm})$ & Pressure $(\mathrm{mT})$ & Source $(\mathrm{W})$ & Bias $(\mathrm{W})$ \\
\hline 13 & 5 & 5 & 10 & 175 \\
\hline \hline
\end{tabular}

illustrates a Ma-N line less than $20 \mathrm{~nm}$ wide and $40 \mathrm{~nm}$ thick standing on a titanium thin film. In order to decrease the resist thickness and thus meliorate the minimal resolution, Ma-N 2401 was diluted in an equivalent volume of anisole. The resist is then $60 \mathrm{~nm}$ thick after spinning and $40 \mathrm{~nm}$ after development. The Ma-D 525 development and subsequent rinsing are critical steps. It is preferable to use an automatic spray developing system and essential to rinse the sample at least $5 \mathrm{~min}$ in stirred deionized (DI) water.

The pattern transfer in the titanium thin film was performed with a multiplex inductively coupled plasma (ICP) SR III-V system from Surface Technology Systems. The samples used are silicon substrates with a thermal oxide underneath a $20 \mathrm{~nm}$ evaporated titanium layer. Table I summarizes the optimized process parameters for the developed $\mathrm{BCl}_{3} / \mathrm{Cl}_{2}$ etching recipe. $\mathrm{A}$ small $\mathrm{BCl}_{3}$ flux was chosen in order to have a good selectivity between the resist and the titanium. The mechanical contribution of $\mathrm{BCl}_{3}$ to the etch process is thus decreased while minimum $\mathrm{Ti}$ sidewall passivation is maintained for better $\mathrm{CD}$ control. ${ }^{5}$ The $\mathrm{Cl}_{2}$ flux was chosen in order to have acceptable etch rates for 20 to $50 \mathrm{~nm}$ thick $\mathrm{Ti}$ film. An increased amount of $\mathrm{Cl}_{2}$ would have led to an increase of the $\mathrm{Ti}$ etch rate $\mathrm{r}^{3,10}$ and thus to a fast and uncontrolled etching procedure. The bias power has been decreased to the minimum in order to decrease the titanium etch rate as well as the resist etch rate and maintain a good selectivity. Lower biases have shown nonuniform etching over the sample. Finally, a $175 \mathrm{~W}$ source power resulted in $40 \mathrm{~nm} / \mathrm{min} \mathrm{Ti}$ etch rates and thus was preferred to higher source power values that showed higher etch rates $(80 \mathrm{~nm} / \mathrm{min}$ for $250 \mathrm{~W})$.

The graphic of Fig. 2 represents the amount of etched material as a function of time for titanium (24 and $40 \mathrm{~nm}$ thick

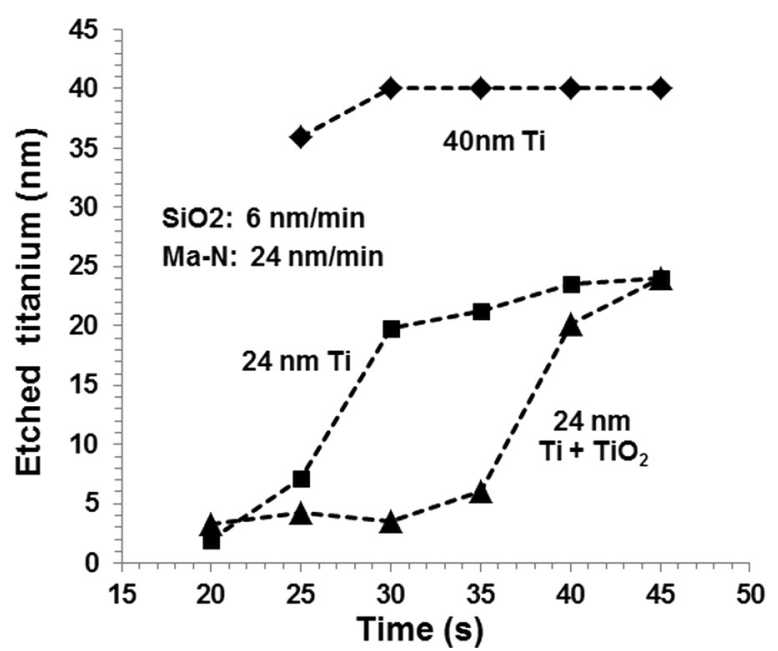

FIG. 2. Etched titanium as a function of time for 40 and $24 \mathrm{~nm}$ thick titanium films, and $24 \mathrm{~nm}$ thick plasma oxidized films $\left(6 \mathrm{~nm}\right.$ of grown $\left.\mathrm{TiO}_{2}\right)$. The $\mathrm{Ma}-\mathrm{N}$, and $\mathrm{SiO}_{2}$ etch rates are given for comparison. films) and $24 \mathrm{~nm}$ thick plasma oxidized Ti. The Ma-N and $\mathrm{SiO}_{2}$ etch rates are given in the graphic for comparison. The amount of etched material has been measured by ellipsometry on samples without resist and/or patterns. The oxidized titanium has been obtained by an oxygen plasma oxidation of a $24 \mathrm{~nm}$ thick titanium sample which led to an increase of $\mathrm{TiO}_{2}$ from less than $2 \mathrm{~nm}$ of native oxide up to $6 \mathrm{~nm}$ of grown oxide. The graphic demonstrates that the titanium oxide has a strong influence over the etching and can act as a masking layer. ${ }^{3}$ Indeed, when only the native oxide lies on top of the Ti film, the etching becomes effective before $20 \mathrm{~s}$ of process, while for the oxidized sample at least $35 \mathrm{~s}$ are necessary for the complete removal of the $\mathrm{TiO}_{2}$ layer. When the titanium layer has been uncovered the etch rate reaches $200 \mathrm{~nm} / \mathrm{min}$ for both samples. An overetch of $10 \mathrm{~s}$ is then mandatory to obtain a silicon oxide surface completely free of Ti residues. These residues are not observed by ellipsometry because of their nanometric dimensions and sparse dispersion but they are clearly visible on SEM images.

Even though the titanium layer is only $24 \mathrm{~nm}$ thick and would theoretically take about $6 \mathrm{~s}$ to etch, a procedure of 35 $\mathrm{s}$ is necessary due to the time needed to remove the Ti native oxide at the beginning of the process and the Ti residues at the end. This effect is clearly highlighted with the $40 \mathrm{~nm}$ layer that needs almost the same etching time as samples half as thick. Argon could have been added to the plasma chemistry in order to increase the physical etching; however, this increases the sidewall roughness and reduces the resist selectivity and is thus not suitable for patterns less than 30 nm. Figure 3 shows SEM images of isolated Ti lines and trenches that have been successfully transferred in the metal through a $45 \mathrm{~s}$ process with the etching parameters described in Table I.

\section{B. Chemical mechanical planarization}

A simplified scheme of the CMP fabrication process is given in Fig. 4. After oxidation of a (1 $\left.00 \begin{array}{ll}1 & 0\end{array}\right)$ silicon substrate, trenches were patterned using EBL followed by plasma etching (step 1 to 3 of Fig. 4). The dimensions of the structures are limited by the EBL resolution and the verticality of the oxide etching recipe. The EBL was carried out using ZEP520A electroresist that has been diluted in anisole in a 2.4 volume ratio for improved resolution. The thickness of

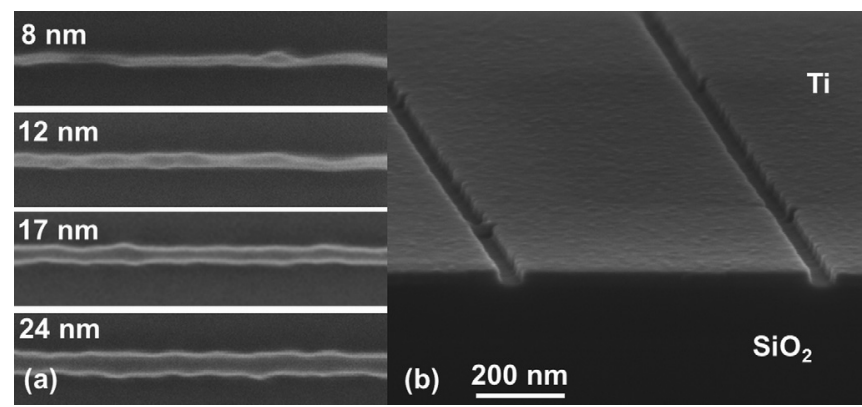

FIG. 3. SEM images of patterned titanium nanostructures: (a) 8, 12, 17, and $24 \mathrm{~nm}$ wide titanium isolated nanowires fabricated with $\mathrm{Ma}-\mathrm{N}$ resist; (b) etched titanium trenches using ZEP resist. 


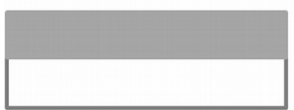

1. oxidation

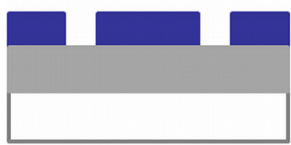

2. e-beam litho

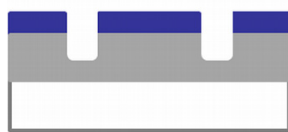

3. ICP etching

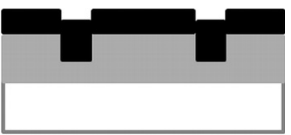

4. Ti evaporation

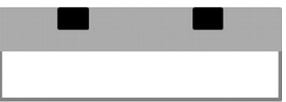

5. CMP

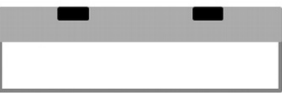

6. CMP
FIG. 4. (Color online) Simplified schematic of the CMP fabrication process.

the resist spun at $5000 \mathrm{rpm}$ decreases from $300 \mathrm{~nm}$ down to $90 \mathrm{~nm}$ for the diluted solution. The EBL was performed at $20 \mathrm{keV}$ with development in O-xylene at room temperature. A $125{ }^{\circ} \mathrm{C}$ post-bake was done in a stove for $30 \mathrm{~min}$ in order to increase the etch resistance and improve the line edge roughness (LER). ${ }^{15}$ The nanoscale patterns were then successfully transferred in the oxide through a $\mathrm{CF}_{4}$ based ICP dry etching. The platen temperature was decreased down to $-20{ }^{\circ} \mathrm{C}$ for improved LER, while the source and bias power were fixed to 100 and $50 \mathrm{~W}$, respectively with a chamber pressure as low as $8 \mathrm{mT}$ in order to increase the sidewalls vertically and the resist/oxide selectivity. This etching recipe together with the adapted EBL permitted the fabrication of very shallow and narrow $(20 \times 20 \mathrm{~nm})$ trenches with almost vertical sidewalls $\left(86^{\circ}\right)$, and a line edge roughness $(3 \sigma)$ of less than $1 \mathrm{~nm} .{ }^{15}$ A titanium film was then deposited by electron beam evaporation in a way to completely fill the oxide trenches. Finally, a planarization step is used to eliminate the residual titanium on the oxide surface and give rise to the embedded titanium nanostructures (step 4 and 5 of Fig. 4).

The titanium removal rate (RR) as well as the silicon dioxide RR depend on many CMP parameters such as the head and platen rotation speeds, the polishing pad and subpad materials, and the amount of slurry. For the fabrication of the devices one wants to remove the silicon dioxide at the same rate as the titanium in order to be able to decrease the metal thickness down to few nanometers (step 5 to 6 of Fig. 4). The easiest way to adapt both material removal rates (MRRs) is to modify the slurry chemistry and/or the nanoparticle density. The original slurry used is a commercial solution from Allied High Tech Products, Inc. with $50 \mathrm{~nm}$ silica nanoparticles diluted in a basic solution. Figure 5 graphic shows the titanium and silicon dioxide material removed as a function of time. The original slurry showed a MRR (solid line in Fig. 5) three times higher for the oxide compared to the titanium.

The first option explored to adjust both MRRs was to dilute the slurry with DI water. The dashed lines of Fig. 5 show that with a dilution of one volume of slurry solution in five volumes of DI water the $\mathrm{SiO}_{2} \mathrm{RR}$ was drastically reduced compared to titanium. The nanoparticle density in the diluted

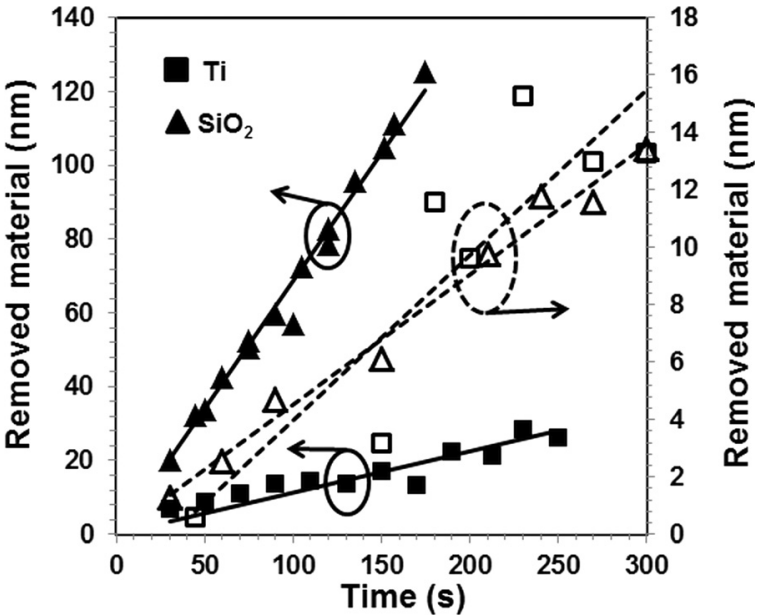

FIG. 5. $\mathrm{SiO}_{2}$ (triangular dots) and $\mathrm{Ti}$ (square dots) removed material as a function of time for a pure slurry (solid lines) and a diluted slurry (dashed lines). The original slurry has been diluted in five equivalent volumes of DI water.

solution is decreased and thus the $\mathrm{SiO}_{2} \mathrm{RR}$, which is more mechanically than chemically driven, is also decreased and a selectivity close to unity was reached. However, the RR has been decreased by a factor of 10 down to less than $3 \mathrm{~nm} / \mathrm{min}$ which is not acceptable for the planarization of layers more than $30 \mathrm{~nm}$ thick. Furthermore, when DI water is added to the slurry, even in a one to one volume ratio, the solution is destabilized and scratches appear on the sample surface. This is explained by micrometric titanium and/or nanoparticle agglomerates that are formed when polishing with the diluted solution. The second proposed option to decrease the $\mathrm{SiO}_{2}$ $\mathrm{RR}$ is to dilute the original slurry in isopropyl alcohol (IPA) instead of DI water. The upper part of the graphic given in Fig. 6 shows the $\mathrm{SiO}_{2}$ / Ti MRR selectivity as a function of slurry dilution. It can be seen that with a dilution of one volume of slurry in 3.5 volumes of IPA the two materials are polished at the exact same rate. With such a solution the MRR has been decreased down to $2.4 \mathrm{~nm} / \mathrm{min}$. For a $100 \mathrm{~nm}$ Ti blanket this would require more than 40 min of polishing.

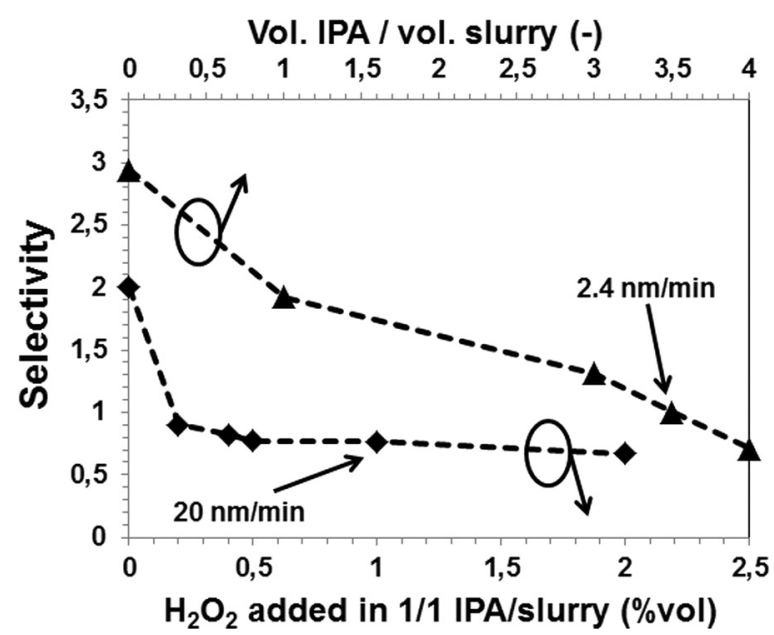

FIG. 6. MRR selectivity as a function of (i) IPA/slurry volume dilution (upper $\mathrm{x}$-axis) and (ii) volume percentage of $\mathrm{H}_{2} \mathrm{O}_{2}(30 \%$ vol.) added in a $1 / 1$ slurry IPA solution (lower $\mathrm{X}$-axis). 


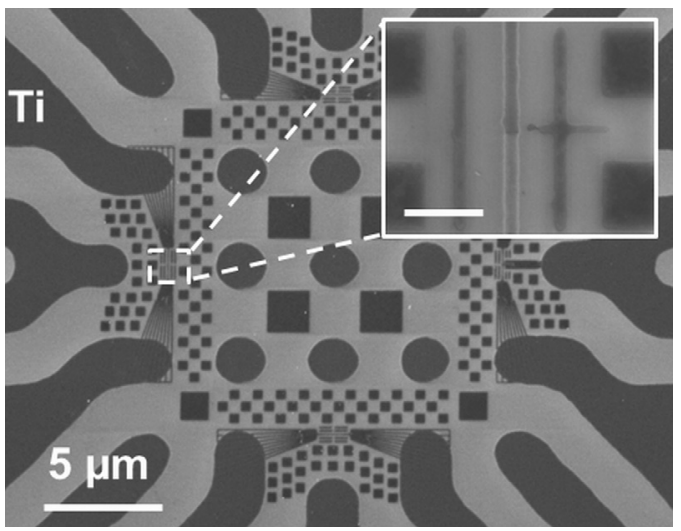

FIG. 7. SEM images of fabricated CMP titanium micro/nanostructures embedded in silicon oxide. The inset is a zoom on the nanostructures (the scale-bar represents $200 \mathrm{~nm}$ ).

The slurry dilution allowed the reduction of the mechanical component of the CMP and thus decreases the MRR by decreasing the nanoparticle density in the solution. The $\mathrm{Ti}$ RR can be modified by changing the chemical component of the CMP. The Ti RR has thus been increased by adding an oxidizer, $\mathrm{H}_{2} \mathrm{O}_{2}$ (30\% vol.), to the slurry solution. The peroxide accelerates the titanium oxidation and as $\mathrm{TiO}_{2}$ is softer than $\mathrm{Ti}$ it is easily removed from the sample surface. The freshly revealed titanium is oxidized and removed mechanically. The in situ oxidation-etching process is repeated during polishing and results in a faster Ti RR. The lower part of the graphic given on Fig. 6 represents the MRR selectivity as a function of volume percentage of $\mathrm{H}_{2} \mathrm{O}_{2}$ added in a $1 / 1$ slurry/IPA solution. The peroxide has a strong effect on the Ti RR at a lower percentage of addition and stabilizes above $0.5 \%$. The oxidation is a self-limited process and is controlled by the rate at which the $\mathrm{TiO}_{2}$ is removed mechanically from the sample surface. The IPA dilution in a volume ratio of 1:1 together with an addition of $1 \%$ of $\mathrm{H}_{2} \mathrm{O}_{2} 30 \%$ vol. showed a $\mathrm{Ti} / \mathrm{SiO}_{2}$ selectivity close to one. The MRR is slightly higher for titanium and is about $20 \mathrm{~nm} / \mathrm{min}$.

Figure 7 shows SEM images of embedded titanium structures. It represents micro and nano-structures emphasizing the fact that both types of structures can be fabricated with a single CMP step. The inset shows a zoomed image of the highlighted nanostructure of a $50 \mathrm{~nm}$ nanowire (NW). The squares and lines on the two images are dummy structures that are mandatory for uniform polishing and MRR both at the device and sample level. With this process we have been able to fabricate NWs $20 \mathrm{~nm}$ wide together with micrometric structures such as $3 \mu \mathrm{m}$ wide electrode lines or squared dummy structures.

When the titanium is completely removed from the silicon dioxide surface and thus only remains in the patterned trenches, further CMP iteration can be used to reduce the structure thickness down to few nanometers (step 5 to 6 of
Fig. 4). Therefore, an adapted CMP end point detection (EPD) method is proposed. The remaining titanium thickness is approximated by in situ NW electrical resistance measurements. The resistance measured is then compared with a material resistivity versus thickness model. ${ }^{16}$ Further CMP iteration then allows the reduction of the titanium trenches thickness down to few nanometers.

\section{CONCLUSION}

This paper presents two different approaches for the fabrication of titanium nanostructures. Both approaches involve an EBL step that defines the lateral dimension of the structures that can be decreased down to $20 \mathrm{~nm}$. With the CMP fabrication process the thickness of the titanium, or any other metal, can be decreased down to few nanometers. With the EBL-plasma etching approach the minimum thickness is; however, limited to $5-10 \mathrm{~nm}$ due to deposition procedure. The planarization process allows the fabrication of micro and nano-structures simultaneously in a single CMP step, though it requires the addition of dummy structures for a uniform planarization.

\section{ACKNOWLEDGMENTS}

This work has been supported by the Natural Sciences and Engineering Resarch Council of Canada (NSERC), Canada, and the Agence Nationale de la Recherche (ANR), France, (ANR-09-BLAN-0411-01).

${ }^{1}$ F. Cardon, Physica (Amsterdam) 27, 841 (1961).

${ }^{2}$ F. Huber, Microelectron. Reliab. 4, 283 (1965).

${ }^{3}$ E. R. Parker, B. J. Thibeault, M. F. Aimi, M. P. Rao, and N. C. MacDonald, J. Electrochem. Soc. 152, C675 (2005).

${ }^{4}$ M. V. S. Ramakrishna, G. Karunasiri, P. Neuzil, U. Sridhar, and W. J. Zeng, Sens. Actuators, A 79, 122 (2000).

${ }^{5}$ S. F. Gilmartin, K. Arshak, D. Bain, W. A. Lane, D. Collins, A. Arshak, B. McCarthy, S. B. Newcomb, and M. Walsh, Microelectron. Eng. 86, 971 (2009).

${ }^{6}$ ITRS Emerging research devices, 2009 edition, http://www.itrs.net.

${ }^{7}$ Q. Xia, W. Robinett, M. W. Cumbie, N. Banerjee, T. J. Cardinali, J. J. Wang, W. Wu, X. Li, W. M. Tong, D. B. Strukov, G. S. Snider, G. Medeiros-Ribeiro, and R. S. Williams, Nano Lett. 9, 3640 (2009).

${ }^{8}$ A. Beaumont, C. Dubuc, J. Beauvais, and D. Drouin, IEEE Electron. Dev. Lett. 30, 766 (2009).

${ }^{9}$ F. Fracassi and R. d'Agostino, Pure Appl. Chem. 64, 703 (1992).

${ }^{10}$ N. M. Muthukrishnan, K. Amberiadis, and A. Elshabini-Riad, J. Electrochem. Soc. 144, 1780 (1997).

${ }^{11}$ D. M. Brown, M. Ghezzo, and J. M. Pimbley, Proc. IEEE 74, 1678 (1986).

${ }^{12}$ M. Krishnan, J. W. Nalaskowski, and L. M. Cook, Chem. Rev. 110, 178 (2010).

${ }^{13}$ J. M. Steigerwald, IEEE Technical Digest of IEDM, 2008, p. 1.

${ }^{14}$ Y.-C. Lee, V. Joshi, A. O. Orlov, and G. L. Snider, J. Vac. Sci. Technol. B 28, C6L9 (2010).

${ }^{15}$ M. Guilmain, A. Jaouad, S. Ecoffey, and D. Drouin, Microelectron. Eng. 88, 2505 (2011).

${ }^{16}$ S. Ecoffey, J.-F. Morissette, N. Jedidi, M. Guilmain, C. Nauenheim, and D. Drouin, Proceedings of the $11^{\text {th }}$ IEEE International Conference on Nanotechnology, 2011, p. 1689. 